\title{
Das Konzept des Verbraucherschutzes in der Machbarkeitsstudie für das Optionale Instrument
}

\author{
Rechtfertigung der Differenzierung des persönlichen Anwendungsbereiches \\ einzelner Normen
}

Fryderyk Zoll

(C) Springer-Verlag 2012

\begin{abstract}
The personal scope of application of the intended Optional Instrument (OI) is limited to business to consumer and business to business contracts. For the former relationship it is always necessary that the business takes the position of the seller or service provider. This article examines the provisions of the Feasibility Study of May 2011 in the light of the question whether the content of the rule itself justifies the narrow scope of application and the pure business or consumer nature of these provisions. In the parts up to performance and non-performance of the contractual obligations the vast majority of the rules is formulated in the style of general civil law provisions. The extension of the scope of application to personal constellations which are not covered by the OI should not be too difficult. In some particular cases there is no sufficient justification for the divergences between the consumer and business provisions. In the part relating to performance and non-performance the differences between the regulation of business to business and business to consumer contracts are wider but also controversial. A unification of these rules is desirable. The extension of the scope of application of the OI is also required in order to ensure the parties' certainty as to the effectiveness of their choice of the instrument. In crossborder contracts it is not easy to determine whether the other party can be qualified as a business or a consumer. Such difficulties would particularly occur in cases of selling platforms. If the parties did not have the security that the adoption of the OI was successful, they would rather
\end{abstract}

Prof. UJ dr hab. Fryderyk Zoll $(\bowtie)$

Katedra Prawa Cywilnego-UJ, Uniwersytet Jagielloński, ul. Bracka 12, 31-005 Kraków, Poland

E-Mail: <fzoll@uos.de>; <fryderyk.zoll@uj.edu.pl> look for other options. Hence, the European legislator should not try to confine the personal scope of application too narrowly. It should be easy to extend it and it is probably necessary for the success of the OI.

\section{Der persönliche Anwendungsbereich des entworfenen Instruments - nur ein Unternehmerrecht?}

Die Verfasser der Machbarkeitsstudie ${ }^{1}$ haben sich dagegen entschieden, ein Instrument des allgemeinen Privatrechts zur Verfügung zu stellen, das unabhängig von der persönlichen Qualifizierung der Vertragsparteien zur Anwendung kommen würde. Die Machbarkeitsstudie enthält zwar noch keine Vorschriften, die den sachlichen und persönlichen Anwendungsbereich eindeutig regeln. ${ }^{2} \mathrm{Um}$ diese wird das Instrument aber in naher Zukunft vervollständigt. ${ }^{3}$ Aus

\footnotetext{
${ }^{1}$ Grundlage dieser Bearbeitung ist die Machbarkeitsstudie von Mai 2011, zu finden unter: <ec.europa.eu/justice/policies/consumer/docs/ explanatory_note_results_feasibility_study_05_2011_en.pdf.> Eine neuere Version von August 2011 ist ebenfalls zu finden (<ec.europa. eu/justice/contract/files/feasibility-study_en.pdf $>$ ) und wurde in den Fußnoten an entsprechender Stelle berücksichtigt. Im Oktober 2011 hat die Kommission auf der Grundlage der Machbarkeitsstudie einen Entwurf für ein Gemeinsames Europäisches Kaufrecht vorgestellt (KOM [2011] 635 final). Die in diesem Beitrag angesprochenen Fragen stellen sich mit gleicher Schärfe für den aktuellen Kommissionsentwurf.

${ }^{2}$ Schulte-Nölke, Scope and Function of the Optional Instrument on European Contract Law, in Schulze/Stuyck, Towards a European Contract Law (2011) 35 (38); siehe auch Gebauer, Optionales Instrument und Internationales Privatrecht, GPR 2011, 157 (157).

${ }^{3}$ Es wurde angedacht, hierfür zunächst sosog „Platzhalter“ vorzusehen, da die Europäische Kommission bislang keine Entscheidung über den Anwendungsbereich des Instruments getroffen hat. Diese Entscheidung behält sich die Kommission noch vor. Vorschläge der Expertengruppe wurden nicht gewünscht. Die Gruppe selbst folgt dem
} 
der Konstruktion der vorgelegten Vorschriften der Studie ergibt sich jedoch eindeutig, dass das geplante Instrument nur dann gewählt werden kann, wenn auf Seiten des Verkäufers bzw. des Dienstleisters ein Unternehmer auftritt. ${ }^{4}$ Der Entwurf steht dann also nicht für Verträge zur Wahl, in denen eine Person als Kunde auftritt, die weder Verbraucher noch Unternehmer ist (zB juristische Personen, die nicht zu gewerblichen Zwecken handeln) sowie dann, wenn keine der Parteien Unternehmer ist (Verträge zwischen Privaten). Darüber hinaus muss auf der Verkäuferseite bzw. der Seite des Dienstleistungserbringers immer ein Unternehmer stehen. Sollte der Verbraucher einem Unternehmer eine Sache verkaufen, kann das Instrument in seiner momentan geplanten Fassung nicht zur Anwendung kommen.

Hinter dieser Entscheidung stehen offensichtlich pragmatische Gründe. Hinsichtlich des Anwendungsbereichs wurden aufgrund der politisch vorgegebenen Knappheit des vorzubereitenden Texts $^{5}$ solche Entscheidungen privilegiert, die eine zu weit gehende Ausdifferenzierung mieden. Aus diesem Grund betrifft der Entwurf nur solche Verträge, welche zwischen zwei oder mehr Unternehmern bzw. Unternehmern und Verbrauchern geschlossen werden. ${ }^{6}$ Dennoch

sog. ,as if"-Ansatz und nimmt bestimmte Entscheidungen der Kommission an, um den Entwurf entwickeln zu können. So: Schulte-Nölke, Scope and Function of the Optional Instrument on European Contract Law, in Schulze/Stuyck, Towards a European Contract Law 35 (36, $38,40 \mathrm{ff})$.

${ }^{4}$ Art 2 Abs 15 und 16 Machbarkeitsstudie (Art 5 Abs 12 und 15 August-Version, obwohl sich hier die Formulierung geändert hat. Definiert wird nur noch „,service provider" und zwar als „seller“ bzw „supplier“. Am Anwendungsbereich wird dies aber nicht ändern.); siehe auch: Einführung zur Machbarkeitsstudie auf der Seite 6 unter: <ec. europa.eu/justice/policies/consumer/docs/explanatory_note_results_ feasibility_study_05_2011_en.pdf>.

${ }^{5}$ Die Expertengruppe stand unter Zeitdruck. Darüber hinaus hatte sie politischen Anforderungen an den Text der Studie gerecht zu werden. So wurde die Gruppe am 26.04.2010 eingesetzt (Entscheidung der Kommission 2010/233/EU). Ihren ersten Entwurf legte sie der Kommission bereits nach knapp einem Jahr, Ende April 2011, vor. Die Machbarkeitsstudie wurde sodann bereits am 03.05.2011 veröffentlicht. Der Auftrag der Gruppe war gemäß Art 4 Abs 5 der Entscheidung 2010/233/EU auf zwei Jahre begrenzt. Zu den Aufgaben der Expertengruppe, siehe: ErwGr 8 sowie Art 2 Entscheidung der Kommission 2010/233/EU vom 26.04.2010, ABl 2010 L 105/110; zu den Anforderungen an den Text siehe: KOM (2010) 348 final - Grünbuch der Kommission vom 01.07.2010, Optionen für die Einführung eines Europäischen Vertragsrechts für Verbraucher und Unternehmen, zu finden unter: <eurlex.europa.eu/LexUriServ/LexUriServ. do? uri=COM:2010:0348:FIN:de:PDF>; siehe auch die Schlussfolgerungen der key stakeholder 2 vom 07.09.2010, zu finden unter: <ec. europa.eu/justice/contract/files/oc-first-meeting_en.pdf>; siehe auch General Introduction des Berichts der Kommission von dem ersten Treffen der Gruppe vom 21.05.2010, zu finden unter: <ec.europa.eu/ justice/contract/files/first-meeting_en.pdf>.

${ }^{6}$ Die Machbarkeitsstudie geht von einem traditionellen Verständnis von Verbraucher und Unternehmer aus. Gemäß Art 2 Abs 1 der Machbarkeitsstudie ist Unternehmer jede natürliche oder juristische Person, die mit einem Zweck handelt, welcher verbunden ist mit ihrem scheinen nicht alle Verträge, die auf Seiten des Verkäufers bzw. des Dienstleistungserbringers durch einen Unternehmer geschlossen werden, von der Studie erfasst zu sein. Der Entwurf sieht keine Regeln für Fälle vor, in welchen auf Seiten des Kunden weder ein Verbraucher noch ein Unternehmer auftritt. ${ }^{7}$ Es sieht so aus, dass zB alle juristischen Personen, welche keine unternehmerische Tätigkeit betreiben, von der Möglichkeit, das Optionale Instrument zu wählen, ausgeschlossen werden sollten. Diese Ausklammerung ist kaum durch das Streben nach einem knappen Text zu rechtfertigen. Sie kann aber die Anwendung des Instruments unnötig erschweren. Die Anwendbarkeit des Instruments hinge so immer von der nicht sicher zu beantwortenden Frage ab, ob die beteiligten Parteien die entsprechende persönliche Eigenschaft innehaben. Die an der Wahl des Instruments interessierte Partei wird die Last der Aufklärung tragen müssen, ob die Gegenpartei unter die erforderliche Kategorie fällt. Der Ausschluss dieser Gruppen aus dem Anwendungsbereich hat zur Folge, dass im Fall unterschiedlicher Verkaufsplattformen, wie beispielsweise E-Bay oder Amazon, die Verwendung des Optionalen Instruments in seiner bislang vorhandenen Form in Frage gestellt werden müsste ${ }^{8}$, weil die richtige Einordnung der Parteien in die durch das Optionale Instrument erfassten Kategorien der Vertragsparteien sehr häufig in einer Grauzone liegt und nicht mit ausreichender Sicherheit festgestellt werden kann. ${ }^{9}$ Als eine Voraussetzung für die Wirksamkeit der Wahl des Optionalen Instruments ist eine persönliche Qualifizierung eher ungeeignet. Für die Sicherheit der Verwendung des Instruments wäre es deswegen wünschenswert keine Kategorien von Personen vom Anwendungsbereich

Handel, ihrem Gewerbe, ihrem Handwerk beziehungsweise Beruf. In der August-Fassung (hier Art 5 Abs 6) wird anstelle von „business“ der Begriff „trader“ verwendet, was keine inhaltliche Änderung zur Folge hat. Allerdings könnte dies eine zusätzliche Schwierigkeit für die Übersetzung in andere Sprachen bedeuten. Nach Art 2 Abs 4 ist jede natürliche Person Verbraucher, die zu einem Zweck außerhalb ihres Handels, Gewerbes, Handwerks beziehungsweise Berufs handelt (jetzt Art 5 Abs 7 August Version). Die Modifikationsversuche die im DCFR unternommen wurden (siehe Art I.-1:105 (3) DCFR), wurden nicht übernommen.

${ }^{7}$ So auch Schulte-Nölke, Scope and Function of the Optional Instrument on European Contract Law, in Schulze/Stuyck, Towards a European Contract Law 35 (41).

${ }^{8}$ Doralt verweist in seinem Beitrag „,Rote Karte oder grünes Licht für den Blue Button?" in AcP 211, 1 (insb 25, aber auch 26, 27) und Fn 90 darauf, dass solche offenen Handelsplattformen bereits ,funktionierende Instrumente" außergesetzlicher Art herausgearbeitet haben, so dass die praktische Bedeutung des Rechtsinstruments, insbesondere in Anbetracht auch der hier aufgeführten Rechtsunsicherheit, schwindet.

${ }^{9}$ So auch Doralt, The Optional European Contract Law and why success or failure may depend on the scope rather than substance, Max Planck Private Law Research Paper No 11/9, 1 (15, 16), zu finden unter: <ssrn.com/abstract=187645>. 
auszuschließen. ${ }^{10}$ In diesem Beitrag soll geprüft werden, ob die Ausgestaltung der Vorschriften der Machbarkeitsstudie dem entgegensteht, den persönlichen Anwendungsbereich auf andere Personengruppen zu erweitern und ob die bereits bestehenden Unterschiede zwischen der Regelung beiderseitiger unternehmerischer Verträge und der Geschäfte mit der Beteiligung von Verbrauchern sachgerecht sind.

\section{Vorvertragliche Pflichten}

\subsection{Inhalt der Informationspflichten}

Nach dem Vorbild der Acquis-Principles und des DCFR regelt die Machbarkeitsstudie vorvertragliche Pflichten sowohl für Verträge zwischen Unternehmern und Verbrauchern als auch für rein unternehmerische Verträge. ${ }^{11}$ In Art 13 wird den Unternehmern zugunsten der Verbraucher eine allgemeine Informationspflicht mit in dieser Vorschrift spezifizierten Inhalten auferlegt. Weiter werden, genauer, Informationspflichten für besondere Vertriebsmodelle bestimmt (für Fernabsatz und für außerhalb von Geschäftsräumen geschlossenen Verträge) ${ }^{12}$, die sich gelegentlich mit der generellen Informationspflicht aus Art 13 decken. ${ }^{13}$ Der Unternehmer soll die Beweislast für die Erfüllung der Informationspflichten tragen. ${ }^{14}$ Die Vorschriften über die vorvertraglichen Informationspflichten im Verhältnis zu Verbrauchern sind zwingend. ${ }^{15}$

\footnotetext{
${ }^{10}$ Für die Ausweitung des personellen Anwendungsbereichs auch auf C2C-Verträge spricht sich Herresthal aus, der mit dem ,,ausgeweitete(n) Wettbewerb der Vertragsrechte“, der „gesteigerte(n) Vertrautheit der Unionsbürger mit den optionalen Regeln“ und „umfangreiche(n) Fallmaterial" argumentiert: Ein europäisches Vertragsrecht als optionales Instrument, EuZW 2011, 7 (10).

${ }^{11}$ Art 13 ff Machbarkeitsstudie (Art 13 ist in der August-Version gestrichen. Die Informationspflichten beginnen mit Art 14); zu den Unterschieden zwischen Acquis-Principles, DCFR und Machbarkeitsstudie siehe näher bei: Beale/Howells, Pre-contractual Information Duties in the Optional Instrument, in Schulze/Stuyck, Towards a European Contract Law 49 (51)

${ }^{12}$ Art 14 ff Machbarkeitsstudie (Art 14 ff August-Version).

${ }^{13}$ Krit im Hinblick auf die Verständlichkeit der nebeneinander geltenden allgemeinen und speziellen sowie Informationspflichten für verschiedene Vertriebsmodelle Jansen in dem noch zu veröffentlichenden Band zu der Tagung „European Law Days“, welche vom 30.06.-01.07.2011 in Osnabrück stattfand; ders ua so bereits auch schon in: Der gemeinsame Referenzrahmen für das Europäische Privatrecht, JZ 2008, 529 (535, 544) mwN; näher zu diesem Konzept: Zoll, Zwei Konzepte des Einbaus vorvertraglicher Informationspflichten in ein System der Vertragsordnung am Beispiel des Entwurfes des Gemeinsamen Referenzrahmens und der Grundregeln des Acquis 1-3 (Der Text wird Anfang 2012 bei MANZ erscheinen).

${ }^{14}$ Art 21 Machbarkeitsstudie (Art 22 August-Version).

${ }^{15}$ Art 22 Machbarkeitsstudie (Art 23 August-Version).
}

Die in Art 23 Abs 1 statuierte Pflicht des Unternehmers, einem anderen Unternehmer Informationen zu erteilen, ist flexibler geregelt. Diese Pflicht trifft den Verkäufer bzw. den Dienstleistungserbringer nur dann, wenn sich das Unterlassen der Informationserteilung nicht mit Treu und Glauben vereinbaren lässt und wenn die Informationserteilung erwartet werden kann. ${ }^{16}$ Abs 2 dieses Artikels nennt präziser die Umstände, die für das Bestehen einer Informationspflicht im Sinne von Abs 1 sprechen. Es gibt keine Vorschrift, gemäß welcher Art 23 zwingend wäre. Der vertragliche Ausschluss kann aber (wenn die weiteren Voraussetzungen vorliegen) der AGB-Kontrolle unterzogen werden. Auch in einem Individualvertrag könnte ein derartiger Ausschluss bedenklich sein, wenn sich eine Informationspflicht aus Treu und Glauben ergeben würde. Da es sich um Informationspflichten vorvertraglicher Art handelt, ist die Frage eines solchen Ausschlusses von geringerer Bedeutung (sie kann sich aber stellen, wenn die Parteien zB vorher eine Rahmenvereinbarung getroffen haben).

Konzeptuell scheint die unterschiedliche Regelung der Informationspflichten in beiden Fallgruppen richtig zu sein. Art 23 liefert ein axiologisches Fundament für das Bestehen einer allgemeinen Informationspflicht. Er verweist auf die Maßstäbe von Treu und Glauben und der legitimen Erwartungen der anderen Partei. In verbraucherrechtlichen Rechtsbeziehungen werden die Anforderungen an Informationspflichten viel präziser und weniger flexibel formuliert, was durch die Schutzbedürftigkeit des Verbrauchers gerechtfertigt wird und einem größeren Grad der Standardisierung von Verbrauchergeschäften dient. ${ }^{17}$

Da Art 23 in bestimmten Bereichen auch Dienstleistungen erfasst, stellt sich die Frage, ob die sich aus der Dienstleistungs-RL ergebenden Informationspflichten ausreichend berücksichtigt werden. Die Dienstleistungs-RL sieht einen umfassenden Katalog von zur Verfügung zu stellenden Informationen vor. ${ }^{18} \mathrm{Im}$ Unterschied zur Machbarkeitsstudie müssen die hier geregelten Informationspflichten unabhängig von der persönlichen Qualifizierung des Kunden erbracht werden. ${ }^{19}$ Das Optionale Instrument darf nicht

\footnotetext{
${ }^{16}$ Art 23 Abs 1 Machbarkeitsstudie (Art 24 August-Version).

${ }^{17}$ Zur Bedeutung von Informationspflichten für Verbraucherverträge siehe: Beale/Howell, Pre-contractual Information Duties in the Optional Instrument, in Schulze/Stuyck, Towards a European Contract Law 49 (49, 53); Gsell in Staudinger, BGB, Eckpfeiler des Zivilrechts, Verbraucherschutz Rz 12, 13 mwN; Ebers, Informations- und Beratungspflichten bei Finanzdienstleistungen, in Schulze/Ebers/Grigoleit, Informationspflichten und Vertragsschluss im Acquis communautaire 171 (174).

${ }^{18}$ Art 22 Dienstleistungs-RL 2006/123/EG, ABl 2006 L 376/36.

${ }^{19} \mathrm{Vgl}$ hierzu den Wortlaut des Art 22 Dienstleistungs-RL („Dienstleistungserbringer“/,Dienstleistungsempfänger“); siehe auch: Schmidt-Kessel in Schlachter/Ohler, Europäische Dienstleistungsrichtlinie - Handkommentar (2008) Vorb zu Art 22 ff Rz 5, Art 22 Rz 3.
} 
unter das Schutzniveau der Richtlinien fallen ${ }^{20}$, auch wenn dort vorgesehenen Maßnahmen als übertrieben erscheinen mögen. ${ }^{21}$

Die Regelung des Art 23 wäre - unabhängig von dem oben herausgearbeiteten Problem der Übereinstimmung mit der Dienstleistungs-RL - auch dann plausibel, wenn solche Vertragsparteien durch den Anwendungsbereich des Optionalen Instruments erfasst wären, die weder als Verbraucher noch als Unternehmer qualifiziert werden können. Die Vorschrift gebietet keine Besonderheiten, die sich aus der unternehmerischen Qualifizierung des Kunden ergeben könnten. Vielmehr betrifft die Regelung auch Vertragssituationen, an welchen sich keine Unternehmer beteiligen. Das flexible System von Art 23 wäre in solchen Fällen gleichermaßen einsatzfähig. Die fehlende Unternehmereigenschaft müsste dann entsprechend bei der Beantwortung der Frage berücksichtigt werden, ob die nach Treu und Glauben zu beurteilenden Umstände vorliegen, die eine Informationspflicht begründen würden.

Würde also der Anwendungsbereich des Optionalen Instruments auf Verträge ohne Beteiligung eines Unternehmers bzw. mit der Beteiligung einer Person, die weder als Verbraucher noch als Unternehmer qualifiziert werden kann, erstreckt, müsste das Konzept der Informationspflichten nicht verändert werden und Art 23 könnte auch in diesen Fällen angewendet werden.

\subsection{Sicherstellung der Richtigkeit der erteilten Information}

Artikel 24 legt der Partei, welche eine Information erteilt, eine Sorgfaltspflicht zur Sicherstellung auf, dass die vor oder bei Vertragsabschluss erteilte Information richtig und nicht irreführend ist. ${ }^{22}$ Für die Verletzung dieser Pflicht gelten die allgemeinen Regeln über die Verletzung einer Informationspflicht. ${ }^{23}$ Diese Vorschrift ist allgemein, folglich ohne Bezug auf die beteiligten Personen formuliert. Im Verhältnis zwischen Unternehmern und Verbrauchern ist sie

\footnotetext{
${ }^{20}$ So auf 1 (Introduction) des Berichts der Kommission von dem vierten Treffen der Gruppe vom 01.-02.09.2010, zu finden unter: <ec. europa.eu/justice/policies/consumer/docs/cfr_report_10_09_01_02_ en.pdf>; siehe auch: Tonner, Das Grünbuch der Kommission zum Europäischen Vertragsrecht für Verbraucher und Unternehmer-Zur Rolle des Verbrauchervertragsrechts im europäischen Vertragsrecht, EuZW 2010, 767 (769).

${ }^{21}$ Herresthal, Ein europäisches Vertragsrecht als Optionales Instrument, EuZW 2011, 7 (11); zu den Folgen des Unterschreitens des Schutzniveaus siehe: Kunz, Verbraucherschutz beim Internethandel in der Europäischen Union - Status quo und Weiterentwicklung (2010) 216 mit einem Verweis auf Müller, Gefahren einer optionalen europäischen Vertragsordnung - Aktionsplan der EG-Kommission zum Vertragsrecht, EuZW 2003, $683(684,685)$.

${ }^{22}$ Art 24 Abs 1 S 1 Machbarkeitsstudie (Art 29 Nr 1 August-Version).

${ }^{23}$ Art 24 Abs 1 S 2 Machbarkeitsstudie (Art 29 Nr 2 August-Version).
}

allerdings zwingendes Recht. ${ }^{24}$ Die genannte Vorschrift ist wenig geglückt. Vielmehr wäre die simple Regel zu bevorzugen, dass eine unrichtige bzw. irreführende Information keine Information ist und die Erteilung einer solchen Information nicht als Erfüllung der Informationspflicht gesehen werden kann. ${ }^{25}$ Eine vertragliche Vereinbarung, die auch zwischen Unternehmern geschlossen wurde, welche eine Erteilung der unrichtigen oder irreführenden Information als Erfüllung einer Informationspflicht qualifizieren würde, scheint eine äußerst merkwürdige Konstruktion zu sein. Es ist schwierig eine derartige Ausprägung des Grundsatzes der Vertragsfreiheit zu akzeptieren. Die vollunternehmerische Natur eines Rechtsgeschäftes kann nicht ein vertragliches „Recht auf Lüge“ zulassen.

Noch aus einem anderen Grund ist Art 24 eine zweifelhafte Regel. Die Vorschrift kann zur Verwässerung der Informationspflicht führen, wenn die Beachtung der Sorgfalt Bestandteil der primären Pflicht zur Informationserbringung sein sollte. Die (bloße) Beachtung der Sorgfalt bei Erteilung einer unrichtigen Information kann aber nicht zur Annahme der Erfüllung der Informationspflicht führen. Bei einer derartigen Auslegung wäre auch die zwingende Natur dieser Norm kaum verständlich.

Es ist nicht einfach zu beurteilen, inwieweit diese Norm auch für Verträge zwischen Privatpersonen bzw zwischen Unternehmern und Personen ohne Verbraucher- und Unternehmereigenschaft gelten könnte. Bei Art 24 handelt es sich um eine nicht ausgereifte Norm, die in dieser Gestalt keine Gültigkeit erlangen sollte. Ihre Abdingbarkeit in rein unternehmerischen Verträgen ist eine Entscheidung, die man nicht begrüßen kann. Im Übrigen ist diese Norm an die allgemeinen Informationspflichten gekoppelt und kann alle persönlichen Konstellationen betreffen. Im Hinblick auf ihren persönlichen Anwendungsbereich ist diese Norm (abgesehen von der Frage der Abdingbarkeit) nicht reformbedürftig. Es gibt aber andere Gründe, die für ihre dringende Abänderung sprechen würden.

\subsection{Rechtsbehelfe im Fall der Verletzung von Informationspflichten}

Die Verletzung von Informationspflichten kann unterschiedliche Sanktionen nach sich ziehen. Eine falsche Information über die Eigenschaft einer Sache könnte beispielsweise die Haftung wegen Vertragswidrigkeit auslösen. Würde durch die Verletzung der Informationspflicht ein Irrtum verursacht werden, könnten unterschiedliche Regeln über Willensmängel zum Einsatz kommen. Steht einem Verbraucher ein Widerrufsrecht $\mathrm{zu}$, wird die Widerrufsfrist entsprechend

\footnotetext{
${ }^{24}$ Art 24 Abs 2 Machbarkeitsstudie (Art 29 Nr 3 August-Version).

${ }^{25}$ Eine solche Regelung ist in Art 2:204 ACQP zu finden.
} 
verlängert. ${ }^{26}$ Neben diesen allgemeineren Rechtsbehelfen sieht die Machbarkeitsstudie besondere Sanktionen für die Verletzung von Informationspflichten vor. Wenn der Vertrag trotz der Verletzung einer Informationspflicht geschlossen wurde und durch das Fehlen der richtigen Information eine legitime Erwartung über das Bestehen einer Verpflichtung auf Seiten des Unternehmers begründet wurde, würde dieser tatsächlich mit einer derartigen Pflicht belastet werden. ${ }^{27}$ Darüber hinaus könnte der Kunde auch einen Schadenersatz geltend machen, vorausgesetzt, dass der Vertrag gar nicht oder mit einem anderen Inhalt geschlossen worden wäre, wenn die Informationspflicht richtig erteilt worden wäre. ${ }^{28}$ Absatz 3 bestimmt ausdrücklich, dass diese Rechtsbehelfe der Verlängerung der Widerrufsfrist sowie der Geltendmachung der Rechte, die im Fall eines Irrtums bzw. einer arglistigen Täuschung greifen, nicht entgegenstehen. In Verträgen zwischen Unternehmern und Verbrauchern können die Rechte aus Art 25 nicht ausgeschlossen bzw. abgeändert werden. ${ }^{29}$ Würde der persönliche Anwendungsbereich des Optionalen Instruments erweitert werden, gäbe es keinen Grund, die Regeln über die Abdingbarkeit dieser Vorschrift abzuändern, wenn der inneren Logik des Optionalen Instruments gefolgt würde. Am Rande soll nur angedeutet werden, dass die Schranken der Vertragsfreiheit auch für rein unternehmerische Verträge überdacht werden müssten. Würde eine Informationspflicht entstehen, die nicht von den Parteien ausgeschlossen wurde, könnte der vollständige Ausschluss der Sanktionen für die Verletzung von Informationspflichten zu nicht kohärenten Lösungen führen. Abgesehen davon, müsste ein Haftungssauschluss dann untersagt werden, wenn er als sittenwidrig anzusehen ist. Das ist aber ein generelles Problem der Machbarkeitsstudie, weil auf die Festlegung der absoluten Grenzen der zulässigen Vertragsgestaltung verzichtet wurde. In vielen Fällen wird dies vermutlich zu einer Quelle zahlreicher Anwendungsschwierigkeiten werden.

Die Regelung der Sanktionen für den Bruch von den Informationspflichten steht einer Erweiterung des persönlichen Anwendungsbereiches im Ergebnis nicht entgegen.

\subsection{Elektronische Verträge}

Die Verfasser der Machbarkeitsstudie sind im Fall von Verträgen, die auf elektronischem Wege geschlossen werden, bei dem vertrauten Modell der E-Commerce-RL 2000/31/ EG geblieben. Das betrifft sowohl die Erteilung der überwiegend zum technischen Vorgang des Vertragsabschluss

\footnotetext{
${ }^{26}$ Art 42 Abs 3 Machbarkeitsstudie (Art 43 Nr 2 August-Version).

${ }^{27}$ Art 25 Abs 1 Machbarkeitsstudie (nicht mehr in der August-Version).

${ }^{28}$ Art 25 Abs 2 Machbarkeitsstudie (Art 30 Nr 1 August-Version).

${ }^{29}$ Art 25 Abs 4 Machbarkeitsstudie (Art 30 Nr 4 August-Version).
}

nützlichen Informationen ${ }^{30}$ wie auch die Verpflichtung zur Korrektur der Eingabefehler ${ }^{31}$ und ebenso die Verpflichtung zur Eingangsbestätigung der Offerte und Annahme ${ }^{32}$. Diese Informations- und Bestätigungspflichten treffen den Unternehmer, der elektronische Mittel zum Abschluss von Verträgen, die keiner individuellen Kommunikation bedürfen, zur Verfügung stellt. ${ }^{33}$ Diese Pflichten sind in Verträgen zwischen Unternehmern und Verbrauchern zwingend ${ }^{34}$, was auch der Regelung der Richtlinie entspricht ${ }^{35}$.

Die E-Commerce-RL sieht keine Einschränkung des persönlichen Anwendungsbereiches auf Seiten des Kunden vor. Die Verbrauchereigenschaft spielt nur für die Unabdingbarkeit der genannten Pflichten eine Rolle. ${ }^{36}$ Die Erweiterung des Anwendungsbereiches auf Kunden, die weder Unternehmer noch Verbraucher sind, würde damit keinen Anpassungsbedarf der vorgeschlagenen Normen auslösen.

Die Erweiterung des Anwendungsbereiches des Optionalen Instruments auf Verträge zwischen Privatpersonen würde keine Veränderung der vorgeschlagenen Vorschriften erforderlich machen. Die Vorschriften über den elektronischen Geschäftsverkehr sind auf Bedürfnisse der Massengeschäfte zugeschnitten. Das in Art 26 vorgesehene Pflichtenprogramm erfasst die notwendige Individualkommunikation ohnehin nicht. Verwenden Privatpersonen von Unternehmern zur Verfügung gestellte Handelsplattformen, könnten diese Unternehmer gemäß Art 24 Abs 1 die Verantwortung für die Erfüllung dieser Pflichten tragen, auch wenn sie nicht Vertragspartei geworden sind. Das würde sich aus der Formulierung von Art 26 Abs 1 ergeben, der die Pflichten nicht notwendigerweise der Vertragspartei, sondern derjenigen Person auferlegt, die die Mittel zum Abschluss des elektronischen Vertrages zur Verfügung stellt. Im Anwendungsbereich, der höchstwahrscheinlich der heutigen Fassung der Machbarkeitsstudie entsprechen wird, wäre auch denkbar, dass es sich in Art 26 Abs 1 um einen Dritten handelt, der mit den Vertragsparteien dadurch verbunden ist, dass er die technische Infrastruktur bereitstellt. Es ist gut vorstellbar, dass diese Plattform den Parteien unter der Voraussetzung zur Verfügung gestellt wird, sich dem mittels dieser Platt-

\footnotetext{
${ }^{30}$ Art 10 Abs 1 lit a E-Commerce-RL; Art 26 Abs 3 lit a Machbarkeitsstudie (Art $25 \mathrm{Nr} 3$ Abs 1 August-Version).

${ }^{31}$ Art 10 Abs 1 lit c E-Commerce-RL; Art 26 Abs 3 lit c Machbarkeitsstudie (Art $25 \mathrm{Nr} 3$ Abs 3 August-Version)

${ }^{32}$ Art 11 Abs 1 E-Commerce-RL; Art 26 Abs 5 der Machbarkeitsstudie (Art $25 \mathrm{Nr} 5$ August-Version).

${ }^{33}$ Art 26 Abs 1 Machbarkeitsstudie (Art 25 Nr 1 August-Version).

${ }^{34}$ Art 26 Abs 7 der Machbarkeitsstudie (Dieser Absatz wurde gestrichen; eingefügt wurde eine Regelung für Fernabsatzverträge, Art 26 August-Version).

${ }^{35}$ Siehe ErwGr 55 E-Commerce-RL.

${ }^{36}$ Art 10 Abs 1 lit a und c, Art 11 Abs 1 E-Commerce-RL $(, \ldots$ außer im Fall abweichender Vereinbarungen zwischen Parteien, die nicht Verbraucher sind...").
} 
form geschlossenen Vertrag und damit dem Optionalen Instrument zu unterwerfen. Diese Auslegung würde auch die Erweiterung des sachlichen Anwendungsbereiches bedeuten und zugleich zur Einbeziehung Dritter führen.

Die Erweiterung des persönlichen Anwendungsbereiches auf Verträge zwischen Privaten und Unternehmern und Personen, die weder Unternehmer noch Verbraucher sind, würde im Hinblick auf Verträge, die unter Zuhilfenahme elektronischer Mittel geschlossen werden, keiner Veränderung der Vorschriften der Machbarkeitsstudie bedürfen.

\subsection{Verpflichtung zur Führung der Verhandlungen nach} Treu und Glauben und zur Achtung der Geheimnisse

Die in Art 27 und 28 enthaltenen Normen gehören zum allgemeinen Privatrecht und sind unabhängig von der Verbraucher- oder Unternehmereigenschaft der Parteien anwendbar ${ }^{37}$. Die grundsätzliche Freiheit, auf den Abschluss des Vertrages zu verzichten, auch wenn zuvor Vertragsverhandlungen geführt wurden, gehört zu den fundamentalsten Bestandteilen der Vertragsfreiheit. ${ }^{38}$ Die Machbarkeitsstudie bestimmt allerdings, dass die Verhandlungen gemäß den Grundsätzen von Treu und Glauben geführt werden müs$\operatorname{sen}^{39}$ und dass die Verletzung dieser Pflicht zu einer Schadensersatzhaftung führen $\mathrm{kann}^{40}$. Die Pflicht, nach Treu und Glauben zu verhandeln, kann nicht vertraglich abbedungen werden, unabhängig davon, in welcher persönlichen Konstellation der Vertrag geschlossen wird. ${ }^{41}$ Würde man über den bisher geplanten persönlichen Anwendungsbereich des Optionalen Instruments hinausgehen, bestünde hier kein Handlungsbedarf. Am Rande soll nur auf die Auffassung von Zimmermann und Jansen hingewiesen werden, die die Regeln über die Haftung wegen des Eintritts in Vertragsverhandlungen, ohne die Absicht den Vertrag abzuschließen, mit dem Recht des Verbrauchers, den Vertrag in bestimmten Fällen frei widerrufen zu können, als unvereinbar gesehen haben. ${ }^{42}$ Tatsächlich stellt sich die Frage, ob ein Verbraucher, der einen Vertrag geschlossen hat, der binnen einer bestimmten Frist widerrufen werden kann, sich haftbar

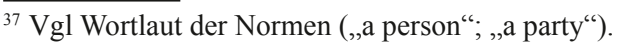

${ }^{38}$ Schulze in Schulze ea, Bürgerliches Gesetzbuch ${ }^{6}$ (2009), Vorbemerkung zu $\S \S 311-319$ Rz 6; Gehrlein/Sutschet in Bamberger/Roth, Beck'scher Online Kommentar ${ }^{20}$ BGB (01.03.2011) § 311 Rz 2; Stadler in Jauernig, Bürgerliches Gesetzbuch ${ }^{13}$ (2009) § 311 Rz 3; Löwisch in Staudinger, BGB (2005) § $311 \mathrm{Rz} 1$.

${ }^{39}$ Art 27 Abs 2 Machbarkeitsstudie (nicht mehr in der August-Version).

${ }^{40}$ Art 27 Abs 3 der Machbarkeitsstudie (nicht mehr in der August-Version).

${ }^{41}$ Art 27 Abs 2 S 2 der Machbarkeitsstudie (nicht mehr in der AugustVersion)

${ }^{42}$ Jansen/Zimmermann, Grundregeln des bestehenden Gemeinschaftsprivatrechts?, JZ 2007, 1113 (1123)
}

machen könnte, wenn er von vornherein den Vertrag zu widerrufen beabsichtigte und somit keinen echten Willen hatte, in die Vertragsbeziehung endgültig einzutreten. Es kann nicht ausgeschlossen werden, dass in der vorgelegten Fassung der Machbarkeitsstudie die Ausübung des Widerrufsrechts durch einem Verbraucher anhand der Maßstäbe von Treu und Glauben bewertet werden kann. Das könnte mit dem sich aus den Richtlinien ergebenden Unionsrecht unvereinbar sein. ${ }^{43}$ Diesbezüglich besteht somit Klärungsbedarf. Es könnte auch bedeuten, dass ein Verbraucher in Fällen, in denen ihm ein Widerrufsrecht zusteht, Verhandlungen abbrechen kann ohne haftbar gemacht zu werden, während ein solches Verhalten für Parteien, die nicht Verbraucher sind, einen Versto $ß$ gegen Treu und Glauben bedeuten würde. $\mathrm{Zu}$ diesem Ergebnis kann man auch durch richtlinienkonforme Auslegung des Optionalen Instruments gelangen ${ }^{44}$ Das sollte aber im Laufe des Rechtsetzungsverfahrens klarer zum Ausdruck gebracht werden.

\section{Vertragsschluss}

Die Vorschriften über den Vertragsschluss werden in der Machbarkeitsstudie personenneutral gefasst. Dem Richtlinienrecht folgend wird lediglich die Vorschrift über die Zusendung unbestellter Waren verbraucherspezifisch formuliert und als solche für zwingend erklärt. ${ }^{45}$

Es gibt auch eine auf die Erklärung des Unternehmers gerichtete Auslegungsregel, die bestimmt, unter welchen Umständen öffentliche Äußerungen über die Lieferung von Waren zu einem bestimmten Preis als eine Offerte zu deuten sind. ${ }^{46}$ Diese Norm ist im Hinblick auf den persönlichen Anwendungsbereich zu eng gefasst. Das Problem öffentlicher Äußerungen, wie zB ein in einer Zeitschrift veröffentlichtes Inserat über die Absicht des Verkaufs eines Privatwagens zu einem bestimmten Preis, muss dann mit einer

\footnotetext{
${ }^{43} \mathrm{Vgl}$ bspw die Formulierung in Art 5 Haustürwiderrufs-RL 85/577/ EWG oder Art 6 Fernabsatz-RL 97/7/EG, siehe auch Art 9 Verbraucherrechte-RL 2011/83/EU. Hier wird das Widerrufsrecht nicht durch die Grenzen von Treu und Glauben relativiert.

${ }^{44}$ Würde das Optionale Instrument als Verordnung erlassen werden, würde es im technischen Sinne keiner richtlinienkonformen Auslegung unterliegen. Nicht zu bezweifeln ist aber, dass sich aus der Funktion des Optionalen Instruments ergibt, dass seine Regelungen nicht unter dem Richtlinienstandard liegen können. Nicht vorstellbar ist nämlich, dass die Union selbst ein Instrument zur Umgehung eigener Mindeststandards entwickelt. Aus diesem Grund muss es praktisch zu einer richtlinienkonformen Auslegung der Vorschriften des Optionalen Instruments kommen. Darüber hinaus könnte auch das Optionale Instrument zur Auslegung von Richtlinien herangezogen werden, so auch Stürner in Tagungsband „European Law Days“ Osnabrück, 30.06.-01.07.2011 (in Druck).

${ }^{45}$ Art 9 Fernabsatz-RL 97/7/EG; Art 39 Abs 1 und 2 Machbarkeitsstudie

${ }^{46}$ Art 30 Abs 3 Machbarkeitsstudie (Art 32 Nr 3 S 2 August-Version).
} 
entsprechenden Regel gelöst werden. In einem solchem Fall wäre es auch empfehlenswert, solche Äußerungen eher als eine Einladung zur Abgabe einer Offerte zu betrachten. Die Feststellung, ob der Vorrat ausreichen würde, bleibt außerhalb der Kontrolle der anderen Partei und aus diesem Grund hängt die Verbindlichkeit einer solchen Äußerung ohnehin von dem guten Willen der Erklärenden ab. Die Umkehrung dieser Regel würde die Erweiterung des persönlichen Anwendungsbereiches zudem vereinfachen, da erfahrungsgemäß davon auszugehen ist, dass sich Privatpersonen trotz ihrer Erklärung bestimmte Spielräume noch offen halten möchten.

Die Machbarkeitsstudie geht davon aus, dass Schweigen keine Annahme der Offerte darstellt. ${ }^{47}$ Von dieser Regel gibt es praktisch keine Ausnahmen, abgesehen von den Fällen der Annahme ohne Zugangserfordernis. ${ }^{48}$ Dies stellt aber keinen echten Fall des Schweigens als Annahme dar, da der Adressat eine bestimmte Handlung vornehmen muss. Die modifizierte Annahme nach Art 37 kann auch als stillschweigende Annahme seitens des Offerierenden gesehen werden. Dies ist allerdings eine eher theoretische Konstruktion, weil das Verfahren zum Abschluss des Vertrages in diesem Fall auch ohne die Fiktion des Schweigens als Annahme erklärt werden kann. ${ }^{49}$ Abgesehen von diesen Fällen nennt die Studie keine weiteren Beispiele der stillschweigenden Annahme. Das ist deswegen verständlich, weil durch den Abschluss des Vertrages im Regelfall auch das Optionale Instrument gewählt wird. Diese grundsätzliche Ablehnung des Schweigens als Annahme macht das ganze System geeignet für das allgemeine Zivilrecht, das unabhängig von dem Status der beteiligten Personen wirkt. Es gibt zwar Rechtsordnungen, wie z. B. das polnische Recht, die den Widerruf der Offerte ${ }^{50}$ bzw. die modifizierende Annahme nur in rein unternehmerischen Beziehungen zulassen. ${ }^{51}$ Diese interne Differenzierung des Verfahrens zum Abschluss des Vertrages nach dem Status der beteiligten Personen leidet allerdings an schwachen teleologischen Grundlagen und bringt unnötige Verwirrung in das System.

Die eventuelle Erweiterung des persönlichen Anwendungsbereiches wird, abgesehen von den genannten Randinterventionen (im Fall der Auslegungsregel hinsichtlich der an die Öffentlichkeit gerichteten Äußerungen) keine Veränderungen erfordern.

\footnotetext{
${ }^{47}$ Art 33 Abs 2 der Machbarkeitsstudie (Art 35 Nr 2 August-Version).

${ }^{48}$ Art 34 Abs 3 der Machbarkeitsstudie (Art 36 Nr 3 August-Version).

49 Schnyder/Strauch in Honsell (Hg), Kommentar zum UN-Kaufrecht Art 19 Rz 20 mwN; Schlechtriem/Schroeter in Schlechtriem/Schwenzer/Bacher (Hg), CISG-Kommentar ${ }^{5}$ (2008) Art 19 Rz 14.

${ }^{50}$ Siehe Art $66^{2}$ plZGB.

${ }^{51}$ Siehe Art $68^{1}$ plZGB; näher hierzu und mit weiteren Beispielen siehe: Terryn, Contract Formation - An Illustration of the Difficult Interface with National Law and Enforcement, in Schulze/Stuyck, Towards a European Contract Law 65 (67) und Fn 7.
}

\section{Widerrufsrecht}

Das Widerrufsrecht ${ }^{52}$ wird hier traditionell als ein Mittel des Verbraucherschutzes verstanden..$^{53}$ Die Verfasser der Machbarkeitsstudie haben es nicht gewagt, mit dem Widerrufsrecht zu experimentieren und es auch für bestimmte Fälle in Massengeschäften unabhängig von der persönlichen Qualifikation des Kunden zu gewähren (mit Ausnahme des Großhandels). Das soll aber nicht kritisiert werden. Eine solche Erweiterung des Widerrufsrechtes ist in der Lehre höchst umstritten. ${ }^{54}$ Tatsächlich wurde die Eignung dieser Rechtsinstitution außerhalb des Verbraucherrechts noch nicht ausreichend durchdacht. Da das Optionale Instrument als solches ein Experiment darstellt, ist eine eher konservative Umgangsweise mit einzelnen Rechtsinstituten durchaus verständlich.

Würde der persönliche Anwendungsbereich des Optionalen Instruments erweitert werden, hätte das keinen Einfluss auf die vorgeschlagenen Vorschriften über Widerrufsrechte, die weiterhin auf Verbraucherverträge beschränkt blieben.

\section{Willensmängel}

Die Vorschriften über Willensmängel ${ }^{55}$ (die auch den Tatbestand des Wuchers erfassen ${ }^{56}$ ) sind sehr traditionell formuliert und erinnern an die Kodifikationen, die aus den Zeiten stammen, in welchen von dem Verbraucherrecht keine Rede war. Dementsprechend spielt der Status der Parteien grundsätzlich keine Rolle. Eine Ausnahme betrifft aber die Abdingbarkeit der Vorschriften über den Irrtum. Nach Art 54 Abs $2^{57}$ können die Irrtumsvorschriften in Verbraucherverträgen nicht abgeändert werden. Würde der Anwendungsbereich auf Personen mit anderem Status erstreckt werden, müsste erwogen werden, ob die zwingende Natur der Vorschriften über den Irrtum auch im Fall der Verträge zwischen Privaten und zwischen Unternehmern und Personen ohne Unternehmer - und Verbraucherstellung angeordnet werden müsste. Ob die Vorschriften über den Irrtum in irgendeinem Bereich zwingend sein sollten, hängt vom

\footnotetext{
${ }^{52}$ Art $40 \mathrm{ff}$ der Machbarkeitsstudie (Art 41 August-Version).

${ }^{53} \mathrm{Vgl}$ Wortlaut Art $40 \mathrm{ff}$ Machbarkeitsstudie (bzw Art $41 \mathrm{ff}$ der August-Version) („The consumer has a right to withdraw ..."); siehe auch Eidenmüller, Widerrufsrechte, in Eidenmüller/Faust/Grigoleit/ Jansen/Wagner/Zimmermann, Revision des Verbraucher-acquis 109 (158).

${ }^{54}$ Zur Ausdehnung der Widerrufsrechte auf Unternehmer siehe Eidenmüller, Widerrufsrechte, in Eidenmüller/Faust/Grigoleit/Jansen/Wagner/Zimmermann, Revision des Verbraucher-acquis 109 (158 ff).

${ }^{55}$ Art $45 \mathrm{ff}$ Machbarkeitsstudie (Art $49 \mathrm{ff}$ August-Version).

${ }^{56}$ Art 48 Machbarkeitsstudie (Art 52 August-Version, wobei Abs 2 und 3 gestrichen wurden).

${ }^{57}$ Art $55 \mathrm{Nr} 2$ August-Version.
} 
Zusammenspiel mit den Vorschriften über vorvertragliche Informationspflichten und deren Verletzung sowie den Vorschriften über das Widerrufsrecht ab. Es ist auch ein System vorstellbar, in welchem auf Irrtumsvorschriften fast gänzlich verzichtet wird. Das angenommene Modell der Informationspflichten mit dem eingebauten System der Vertragsanpassung (Art 25 Abs 1) verringert den Bedarf für eine separate Regelung über Willensmängel. Außerhalb des zwingenden Verbraucherrechts müsste diese vielschichtige Struktur sodann bei der AGB-Kontrolle berücksichtigt werden, da ein Ausschluss der Rechte, die im Fall eines Irrtums einer Vertragspartei greifen, für diese nicht notwendigerweise einen Entzug des Schutzes bedeutet, soweit sich auch zufriedenstellende Ergebnisse aus konkurrierenden Grundlagen ergeben. Erlangt das Optionale Instrument Geltung und sind die ersten Erfahrungen gemacht worden, ist zukünftig allerdings zu überprüfen, ob das System der Rechtsinstrumente, die die Korrektheit der Willensbildung gewährleisten sollen, in sich gut abgestimmt ist. Momentan überlappen sich das System der Willensmängel, der Informationspflichten sowie das verbraucherrechtliche Widerrufsrecht auf eine inkohärente Weise. Möglicherweise ist es aber zu früh, diese Widersprüche zu beseitigen. Zudem kann das System auch mit diesen Widersprüchen passabel funktionieren. Dennoch sollten diese Probleme in der Zukunft beseitigt werden.

\section{Bestimmung des Vertragsinhalts}

Im dritten Teil der Machbarkeitsstudie werden die Inhalte geregelt, die unter dem gemeinsamen Titel „Festlegung dessen, was im Vertrag ist" zusammengefasst werden. Es handelt sich hierbei um die Regeln über die Auslegung ${ }^{58}$, den Inhalt und die Wirkung von Verträgen ${ }^{59}$ sowie über missbräuchliche Klauseln ${ }^{60}$.

\subsection{Auslegung}

Die Mehrheit der Vorschriften, die die Auslegung von Verträgen regeln, ist vom persönlichen Status der Parteien unabhängig, zumindest wenn der Wortlaut dieser Vorschriften betrachtet wird. Eine ausdrückliche Ausnahme findet sich in Art 62 $2^{61}$, der in Abs 1 den Grundsatz der verbraucherfreundlichen Auslegung bestimmt und in Abs 2 diese Regel für unabdingbar erklärt. Die Erweiterung des persönlichen Anwendungsbereiches wird keine Notwendigkeit der Abänderung dieser Vorschriften veranlassen.

\footnotetext{
${ }^{58}$ Art $56 \mathrm{ff}$ Machbarkeitsstudie (Art 59 ff August-Version).

${ }^{59}$ Art $64 \mathrm{ff}$ Machbarkeitsstudie (Art $67 \mathrm{ff}$ August-Version).

${ }^{60}$ Art 77 ff Machbarkeitsstudie (Art 80 ff August-Version).

${ }^{61}$ Art 65 August-Version.
}

\subsection{Inhalt und Wirkung von Verträgen}

Im siebten Kapitel des dritten Teils des Optionalen Instruments werden unterschiedliche Normen zusammengetragen, nach welchen der Inhalt eines Rechtsverhältnisses zu bestimmen ist. Der Inhalt ist abzuleiten aus der Vereinbarung der Parteien, die nicht Gegenstand zwingender Regeln des Instruments ist ${ }^{62}$, der Bedeutung der Gewohnheiten und Praktiken ${ }^{63}$ und der Methoden der Ergänzung der Übereinstimmung der Parteien ${ }^{64}$. Darüber hinaus wird die Bedeutung öffentlicher Äußerungen des Unternehmers sowie des Herstellers bzw anderer Personen, die sich in einer Vertriebskette befinden, festgelegt ${ }^{65}$. Darauf folgen die Vorschriften über sog Integrationsklauseln (merger clause) ${ }^{66}$, pactum de forma ${ }^{67}$ sowie die Regeln über Bestimmung des Preises $^{68}$, der Sprache ${ }^{69}$, der Beendigung von Dauerschuldverhältnissen ${ }^{70}$ und die Regeln über die Verträge zugunsten Dritter $^{71}$. Die Mehrheit dieser Bestimmungen ist wiederum „neutral“ formuliert. Die persönliche Qualifikation der Vertragsparteien spielt damit keine Rolle für diese Vorschriften, was sich aus ihrem Wortlaut ergibt. Ausnahmen hierzu sind in Art 67 und $68 \mathrm{zu}$ finden.

Fraglich ist, ob all diese Vorschriften tatsächlich für sämtliche persönliche Konstellationen geeignet sind. So ist zu fragen, ob nicht die Betonung der Bindungswirkung von Gewohnheiten und Praktiken (zumindest) im Fall der Verbraucherverträge (vgl Art 65) zu weit geht. Optimalerweise sollte das Optionale Instrument in unterschiedlichen Mitgliedsstaaten Geltung erlangen. Es besteht daher immer das Risiko, dass durch Art 65 ein bestimmter Inhalt des Vertrages für den Verbraucher überraschend sein kann, insbesondere, wenn Gewohnheiten Geltung erlangen, über welche der Verbraucher nicht informiert wurde. Solche Gefahren könnte auch Art 66 bergen. Artikel 66 bringt eine sehr weitgehende Kompetenz des Gerichts zum Ausdruck, den Inhalt des Vertrages zu gestalten. Das kann auch der Einheitlichkeit der Anwendung des Optionalen Instruments im Wege stehen. Angesichts der Funktion des Optionalen Instruments, eine europaweite Einheitlichkeit der Entscheidungen im Anwendungsbereich herzustellen, sollte zurückhaltender $\mathrm{zu}$ solchen traditionsreichen Konstruktionen

\footnotetext{
${ }^{62}$ Art 64 lit a Machbarkeitsstudie (Art 67 lit a August-Version).

${ }^{63}$ Art 64 lit b, Art 65 Machbarkeitsstudie (Art 67 lit b, Art 66 AugustVersion - ausdrücklich begrenzt auf Unternehmer).

${ }^{64}$ Art 64 lit d, Art 66 Machbarkeitsstudie Art 67 lit d August-Version).

${ }^{65}$ Art 67 Machbarkeitsstudie (Art 70 August-Version).

${ }^{66}$ Art 68 Machbarkeitsstudie (Art 73 August-Version).

${ }^{67}$ Art 69 Machbarkeitsstudie (nicht mehr in der August-Version).

${ }^{68}$ Art 70 Machbarkeitsstudie (Art 75 August-Version).

${ }^{69}$ Art 73 Machbarkeitsstudie (Art 77 August-Version).

${ }^{70}$ Art 74 Machbarkeitsstudie (Art 78 August-Version).

${ }^{71}$ Art 76 Machbarkeitsstudie (Art 79 August-Version).
} 
des Privatrechts gegriffen werden, die sich im nationalen Bereich zwar bewährt haben, aber bei grenzübergreifender Anwendung notwendigerweise zur Rechtsunsicherheit führen müssen.

Personenbezogen ist die Formulierung von Art 67. Die von einem Unternehmer getätigte Äußerung gegenüber der anderen Partei wird unter bestimmten Umständen (lit a und b) Bestandteil des Vertrages (Abs 1). Im Hinblick auf öffentliche Äußerungen ist eine Einschränkung des Anwendungsbereiches dieser Norm auf den Unternehmer verständlich. Im Fall anderer potenzieller Vertragsparteien wäre eine derartige Regel gegenstandslos. Anders ist es mit Äußerungen, die direkt gegenüber der anderen Vertragspartei abgegeben werden. In einem solchen Fall hätte diese Norm einen Sinn, auch wenn der Partei keine Unternehmereigenschaft zukäme. Würde der persönliche Anwendungsbereich des Optionalen Instruments erweitert werden, müsste man erwägen, ob Art 67 Abs 1 im Fall der Äußerungen, die gegenüber der Vertragspartei getroffen werden, auch in Verträgen zwischen Privaten zur Anwendung kommen sollte.

Darüber hinaus besteht ein Widerspruch zwischen Art 67 und Art 106 Machbarkeitsstudie. Nach Art 106 schließt die Kenntnis bzw fahrlässige Unkenntnis des Käufers von der Vertragswidrigkeit zum Zeitpunkt des Vertragsschlusses die Haftung des Verkäufers aus. Diese Vorschrift gilt nur für Verträge zwischen Unternehmern. Für Verbraucherverträge gilt diese Regel indes nicht. Nach Art 67 Abs 1 lit a würde aber $z B$ eine Zusicherung einer bestimmten Eigenschaft durch den Unternehmer nicht Vertragsbestandteil, wenn von dem Verbraucher erwartet werden kann, dass ihm die Unrichtigkeit der Äußerung des Unternehmers bewusst ist. Es fehlt an einer Abstimmung beider Vorschriften. Durch die Einschränkung in Art 106 versuchten die Verfasser, den Verbraucher auch in den Fällen zu schützen, in welchen der Verbraucher die Vertragswidrigkeit sogar kannte. An einer anderen Stelle heißt es aber, dass selbst fahrlässige Unkenntnis des Verbrauchers von der Unrichtigkeit einer bestimmten Aussage des Unternehmers bewirkt, dass diese Aussage irrelevant für den Inhalt des Vertrages ist (Art 67 Abs 1 lit a). Die ausdrückliche Zusicherung einer bestimmten Eigenschaft der Sache würde unter solchen Umständen ohne negative Folgen für den Unternehmer bleiben, was sich aber mit der Idee des Art 106, der die Verbraucher aus seinem Anwendungsbereich ausklammert, nicht vereinbaren lässt. Das Problem liegt darin, dass bei der jetzigen Formulierung des Art 67 Abs 1 auch die Erteilung der vorvertraglichen Informationen erfasst wird und in einem solchen Fall die Ausnahmen aus den Buchstaben a und $\mathrm{b}$ zu nachteilig für den Kunden wären.

Direkt die Verbraucherverträge betrifft Art 67 Abs 3. Nach dem Vorbild der Verbrauchsgüterkauf-RL ${ }^{72}$, aber

\footnotetext{
${ }_{72}$ Art 2 Abs 2 lit d und Abs 4 Verbrauchsgüterkauf-RL 1999/44/EG.
}

auch unter dem Einfluss der einschlägigen Lösungen der Acquis-Principles ${ }^{73}$ und des DCFR ${ }^{74}$ werden die Aussagen der unternehmerischen Vertragspartei sowie öffentliche Äußerungen und die Äußerungen des Herstellers und anderer Personen, die eine höhere Position in der Vertriebskette einnehmen als der unternehmerische Vertragspartner selbst, mit den Aussagen dieses Vertragspartners gleichgestellt. ${ }^{75}$ In den Acquis-Principles sowie im DCFR ist dies keine Verbrauchernorm, sondern eine Norm, die auch alle anderen Kundentypen mit einbezieht. Vorausgesetzt, dass die Norm nicht zwingend sein wird, kann sie tatsächlich über das Verbraucherrecht hinaus gelten. In der heutigen Fassung der Studie wird Art 67 durch seinen Abs 4 in Verbraucherverträgen zwingend, was abgesehen von der oben genannten faktischen Abschwächung des Verbraucherschutzes (Abs 1) grundsätzlich richtig ist.

Art 67 Abs 1 ist so formuliert, als würde der Anwendungsbereich der Studie nicht nur Verträge unter zumindest einseitiger Beteiligung der Unternehmer erfassen. Art 67 Abs 1 ordnet in bedingter Form an, dass diese Vorschrift nur dann zur Anwendung kommt, wenn eine Partei Unternehmer ist. Es scheint so, als würde eine derartige Situation eine Ausnahme darstellen, obwohl sie die einzig mögliche Konstellation sein kann. Würde aber der Anwendungsbereich des Optionalen Instruments auf andere Personen erweitert werden, hätte eine derartige Formel Sinn.

Die zweite Norm dieses Kapitels betrifft sog. Integrationsklauseln (merger clauses). Art 68 Abs 1 regelt die Folgen einer vertraglichen Vereinbarung, gemäß welcher die Vertragsurkunde alle Bestimmungen des Vertrages zum Ausdruck bringt. ${ }^{76}$ In Verbraucherverträgen sollen derartige Vertragsklauseln unwirksam bleiben. ${ }^{77}$

Es ist grundsätzlich richtig, dass eine Integrationsklausel in Verbraucherverträgen keine Wirkung entfalten soll. Im Verbraucherrecht würde eine wirksame merger clause diejenigen Zwecke erreichen, die der Gesetzgeber mit der Auferlegung zahlreicher vorvertraglicher Pflichten auf den Unternehmer zu erreichen versucht. Wenn die berechtigten Erwartungen des Verbrauchers eine wesentliche Rolle für die Feststellung der Unternehmerpflichten spielen sollen, kann der Inhalt des Vertrages nicht nur auf den Inhalt der Vertragsurkunde reduziert werden. Vielleicht aber ist diese

\footnotetext{
${ }_{73}$ Art 4:107, Art 4:108 ACQP.

${ }^{74}$ Art II.-9:102 DCFR; vgl auch IV.A.-2:303 DCFR.

${ }^{75}$ Näher hierzu auch Looschelders/Makowsky, Inhalt und Wirkungen von Verträgen, GPR 2011, 106 (108).

${ }^{76}$ Ausführlicher zur merger clause, ihren Vorteilen und den Gefahren, die eine solche Regelung in sich birgt, Zoll, Uwagi do artykułu 92 projektu - tzw merger clause, Transformacje Prawa Prywatnego 4/2010, 61 (61 f); siehe auch: Meyer, Die privatautonome Abbedingung der vorvertraglichen Abreden - Integrationsklausel im internationalen Wirtschaftsverkehr, RabelsZ 72 (2008) 562 ff.

${ }^{77}$ Art 68 Abs 3 Machbarkeitsstudie (Art 73 Nr 3 August-Version).
} 
absolute Entkräftung der merger clause in Verbraucherverträgen zu weitgehend. Erteilt der Unternehmer die gesetzlich vorgeschriebenen Informationen schriftlich, dürfte die Wirkung dieser Informationen auf den Vertrag sicherlich nicht durch eine Klausel vernichtet werden. Das heißt aber nicht, dass der Unternehmer kein legitimes Interesse daran hat, sich vor Behauptungen zu schützen, dass zB tatsächlich andere Informationen erteilt wurden, als das schriftlich festgelegt wird. In Massengeschäften mit Verbrauchern ist das Interesse des Unternehmers an der Standardisierung des Inhalts von Rechtsgeschäften legitim. Wenn das nicht zur Umgehung der Erfüllung der vorvertraglichen Pflichten führen würde, sollte eine bestimmte Wirkung der merger clause auch in Verbraucherverträgen wirksam bleiben. Auch in diesen Fällen könnte der Verbraucher zusätzlich durch die Klauselkontrolle ausreichend geschützt werden.

Daraus ergibt sich aber auch nicht, dass eine Integrationsklausel im rein unternehmerischen Verkehr ohne Weiteres unbedenklich ist. ${ }^{78}$ Abgesehen von klaren Missbrauchsfällen $^{79}$ sollte eine merger clause zusätzlich in den Fällen nicht greifen, in welchen ein Unternehmer auch gegenüber einem anderen Unternehmer zur Erteilung vorvertraglicher Informationen verpflichtet war. ${ }^{80}$

Die Formulierung der Integrationsklausel sollte neu überdacht werden, um die unterschiedlichen Fallkonstellationen zu erfassen. Es ist nicht zu bestreiten, dass die merger clause im Fall von Verbraucherverträgen besonders gefährlich für die Interessen des Verbrauchers sein könnte. Ein absolutes Verbot ist allerdings selbst in Verbraucherverträgen zu restriktiv und sollte gemildert werden. Auch in sonstigen Verträgen verdient eine differenziertere Vorgehensweise Vorzug, die vor allem in Fällen des Bestehens von vorvertraglichen Informationspflichten die Wirkung der Klausel abschwächt.

\subsection{Missbräuchliche Klauseln und optionale Leistungen}

In Kapitel 8 der Machbarkeitsstudie wird das Recht der missbräuchlichen Klauseln geregelt. Darüber hinaus regelt der Entwurf eine Sonderfrage der Nebenvereinbarungen, die nach der Absicht des Unternehmers dann getroffen werden sollen (und zusätzliche Zahlungsverpflichtungen des Kunden auslösen), wenn der Verbraucher sie nicht abgelehnt hat. ${ }^{81}$

\footnotetext{
${ }^{78}$ So auch Looschelders/Makowsky, Inhalt und Wirkungen von Verträgen, GPR 2011, 106 (108).

${ }^{79}$ Die hier angesprochenen Missbrauchsfälle können nicht auf der Grundlage der Machbarkeitsstudie wirksam bekämpft werden. Die AGB-Kontrolle allein reicht nicht aus. Es fehlen andere Instrumente, die sowohl dem Inhalt des Rechtsgeschäftes als auch der Ausübung des subjektiven Rechts Schranken setzen würden.

${ }^{80}$ Siehe Art 23 Machbarkeitsstudie (Art 24 August-Version).

${ }^{81}$ Art 88 Machbarkeitsstudie (Art $72 \mathrm{Nr} 1$ August-Version).
}

Im Recht der missbräuchlichen Klauseln wird zwischen Verträgen zwischen Unternehmern und Verbrauchern und den rein unternehmerischen Verträgen deutlich differenziert. Es gibt zwar einen kleinen gemeinsamen Teil (Art 77-79 ${ }^{82}$ ). Allerdings unterscheiden sich die Grundstrukturen der Klauselkontrolle auf den ersten Blick sehr deutlich voneinander. Trotz dieser oberflächlichen Divergenz ist das Grundkonzept der Kontrolle ähnlich. In Verbraucherverträgen erfasst die Kontrolle nicht nur die Klauseln, die zur einmaligen Verwendung bestimmt werden, sondern auch solche, die ausgehandelt werden. ${ }^{83}$ Die Tatsache, dass eine Klausel in einem bestimmten Umfang ausgehandelt wurde, gehört zu einem der Umstände, die bei der Prüfung der Missbräuchlichkeit berücksichtigt werden müssen. Um Gegenstand der Kontrolle werden zu können, muss eine Klausel aber von dem Unternehmer vorgeschlagen werden, auch wenn sie dann nachträglich Gegenstand der Aushandlung ist (Art 81 Abs 2). Das ist ein Erfordernis, das dieses System der Kontrolle mit dem Phänomen der AGB verbindet.

Für Verbraucherverträge werden zwei Listen von Klauseln vorgesehen - die schwarze und die graue Liste ${ }^{84}$. Der allgemeine Test zur Überprüfung missbräuchlicher Klauseln wird gestützt auf die Begriffe von Treu und Glauben und das „redliche Handeln“ (fair dealing) ${ }^{85}$. Das System der Kontrolle verzichtet auf eine separate Einbeziehungskontrolle. Stattdessen wird die Frage, ob der Verwender die Aufmerksamkeit des Kunden auf die Klauseln ausreichend geweckt hat, im Rahmen der Inhaltskontrolle zu beantworten versucht. ${ }^{86}$ Ist der Kunde Verbraucher, so wird im Abs 2 ergänzt, dass ein bloßer Hinweis auf die Verwendung der Klauseln nicht genügt, auch dann nicht, wenn der Verbraucher die Urkunde unterzeichnet. Die Norm kommt also grundsätzlich ohne Rücksicht auf den persönlichen Status der Parteien zur Anwendung. Die verbraucherspezifische Ausnahme betrifft nur einen kleinen Aspekt dieser Kontrolle.

Das System der Klauselkontrolle in rein unternehmerischen Verträgen unterscheidet sich von seinem Pendant im

\footnotetext{
${ }^{82}$ Art 80-82 August-Version.

${ }^{83}$ Art 81 Abs 2 der Machbarkeitsstudie (nicht mehr der August-Version vorhanden); krit dazu, dass der Anwendungsbereich der Klauselkontrolle sich nicht nur auf nicht individuell ausgehandelte Klauseln beschränkt: Mazeaud, Unfairness and Non-negotiated Terms, in Schulze/Stuyck, Towards a European Contract Law 123 (127); zu dieser Problematik in den Acquis Principles und im DCFR siehe auch Zoll, Unfair Terms in the Acquis Principles and Draft Common Frame of Reference: A Study of the Differences between the Two Closest Members of One Family, Juridica International XIV/2008, 69 (74 ff).

${ }^{84}$ Art 83 und 84 Machbarkeitsstudie (Art 85 und 86 August-Version).

${ }^{85}$ Siehe Art 81 Abs 1 und Art 85 Abs 1 lit c der Machbarkeitsstudie (Art 84 Nr 1 und Art 87 Nr 1 Abs 2 August-Version); krit hierzu Mazeaud, Unfairness and Non-negotiated Terms, in Schulze/Stuyck, Towards a European Contract Law 123 (128).

${ }^{86}$ Art 86 Abs 1 Machbarkeitsstudie (Art 71 Nr 1 August-Version).
} 
Bereich des Verbraucherrechts in relativ geringem Maße. Anders als es im System des DCFR der Fall is ${ }^{87}$ und vergleichbar mit dem System der Acquis-Principles ${ }^{88}$, erstreckt sich die Kontrolle auch auf die zur einmaligen Verwendung bestimmten Klauseln, die von einer Partei vorgeschlagen werden. ${ }^{89}$ Der Begriff der AGB (standard contract terms) wird nur (mit Relevanz auch für Verbraucherverträge) in Bezug auf die Frage der überraschenden Klausel, bei der Vermutung des Nichtaushandelns in unternehmerischen Verträgen und bei sich widersprechenden Klauseln erörtert. ${ }^{90}$ Bei Verbraucherverträgen ist die Vermutung des Nichtaushandelns allein auf die Verbrauchereigenschaft des Vertragspartners zurückzuführen. ${ }^{91}$ Bei rein unternehmerischen Verträgen ist man nur deshalb bei der Vermutung des Nichtaushandelns der AGB geblieben, weil man keine andere Anknüpfung für diese Vermutung finden konnte. Würde der Anwendungsbereich des Optionalen Instruments auf andere Personen erweitert, müsste die mit den AGB verbundene Vermutung auch auf andere Personenkonstellationen angewendet werden.

Der Test zur Überprüfung missbräuchlicher Klauseln, der auf beidseitig unternehmerische Verträge Anwendung findet, unterscheidet sich von dem für die Verbraucher anwendbaren Verfahren nur durch die Nennung des zusätzlichen Kriteriums der groben Abweichung von den guten Handelspraktiken. ${ }^{92}$ Dieses Kriterium stammt ua aus der Zahlungsverzugs-RL ${ }^{93}$ und wurde auch in das System der Klauselkontrolle in den Texten der Acquis-Principles ${ }^{94}$ und

\footnotetext{
${ }^{87}$ Art II.-9:404 DCFR.

${ }^{88}$ Art 6:301 Abs 2 ACQP; ausf zu den Unterschieden der Klauselkontrolle im DCFR und den Acquis Principles siehe: Zoll, Unfair Terms in the Acquis Principles and Draft Common Frame of Reference: A Study of the Differences between the Two Closest Members of One Family, Juridica International XIV 2008, 69

${ }^{89} \mathrm{Vgl}$ Art $77 \mathrm{ff}$ Machbarkeitsstudie (Art $80 \mathrm{ff}$ August-Version); siehe auch Looschelders/Makowsky, Inhalt und Wirkungen von Verträgen, GPR 2011, 106 (113).

${ }^{90}$ Obwohl in Art 2 Abs 17 (Art 5 Nr 5 August-Version) definiert, taucht der Begriff der „standard terms" nur drei Mal auf und zwar in Art 5 Abs 1, 3, Art 38 und Art 87 der Machbarkeitsstudie auf. In der Version von August wurde Art 87 gestrichen. Der Begriff „standard contract terms" ist in der neuen Version daher - außer in der Definition - nur noch in Art $8 \mathrm{Nr} 3$ und Art 40 zu finden.

${ }^{91} \mathrm{Vgl}$ auch Art II.-9:403 DCFR.

${ }^{92}$ Art 85 Abs 1 lit c Machbarkeitsstudie (Art 87 Nr 1 Abs 2 AugustVersion).

${ }^{93}$ An dieser Stelle hat man sich der Begrifflichkeit aus Art 3 Abs 3 Zahlungsverzug-RL 2000/35/EG und Art 7 Abs 1 lit a Zahlungsverzug-RL 2011/7/EU bedient. Allerdings wird dieser Begriff in der Feasibility Study, wie auch schon in den Acquis Principles und dem DCFR, mit neuem Inhalt gefüllt.

${ }^{94}$ Art 6:301 Abs 2 ACQP; Pfeiffer/Ebers, Art 6:301 Rz 1, in Research Group on the Existing EC Private Law (Acquis Group), Principles of the Existing EC Contract Law (Acquis Principles), Contract II (2009) 319.
}

des DCFR ${ }^{95}$ integriert. ${ }^{96}$ Bei dieser Formulierung handelt sich aber nicht um einen qualitativen Unterschied im System der Klauselkontrolle, sondern lediglich um einen Hinweis für den Richter, dass in beiderseitig unternehmerischen Verträgen die Inhaltskontrolle wesentlich zurückhaltender zur Anwendung kommt. Für Verträge zwischen Unternehmen sieht die Machbarkeitsstudie keine Listen verbotener Klauseln vor. Es ist aber zu erwarten, dass auch im unternehmerischen Bereich die für Verbraucher geltenden Listen bei der Ausfüllung der Generalklausel der Klauselkontrolle nicht unbeachtlich bleiben. ${ }^{97}$

Die Verfasser der Machbarkeitsstudie haben im Bereich der Klauselkontrolle ein System geschaffen, das einen relativ weitgehenden Eingriff in den Vertrag rechtfertigt. Das Verbraucher- und Unternehmerrecht werden nach grundsätzlich ähnlichen Prinzipien geordnet. ${ }^{98}$ Das Phänomen der Standardverträge, die die mehrmalige Verwendung bestimmter Klauseln beinhalten, wird nicht als eine erforderliche Rechtfertigung des Eingriffs in die Gestaltungsfreiheit der Parteien benötigt. Eine Erweiterung des Systems auf die Kunden, die weder Verbraucher noch Unternehmer sind, würde die Logik eines solchen Systems nicht brechen. Für die Zwecke der Machbarkeitsstudie sollte vielleicht eher das „Unternehmermodell“ verwendet werden, das etwas mehr Flexibilität erlaubt. Würden auch Verträge zwischen privaten Personen unter den Anwendungsbereich des Optionalen Instruments gefasst, wäre das System der Klauselkontrolle inadäquat und eine zu weitgehende Einschränkung der Vertragsfreiheit nicht erforderlich.

Es stellt sich aber die generelle Frage, ob dieses System, das eigentlich alle Verträge - bis auf geringe Ausnahmen - der Inhaltskontrolle unterstellt, ohne wesentliche Schwellen für die Zulässigkeit dieser Kontrolle aufzubauen ${ }^{99}$, dem Richter nicht eine zu große Macht einräumt und in einem gesamteuropäischen Raum zu einer zu großen Unsicherheit führt. Fraglich ist, ob das Modell der AGB-Kontrolle nicht

\footnotetext{
${ }_{95}$ Art II.-3:101 (2) DCFR; v. Bar/Clive, Principles, Definitions and Model Rules of European Private Law, Draft Common Frame of Reference (DCFR), Full Edition (2009) Comment zu Art III.-3:101, 202.

${ }^{96}$ Zoll (Fn 88), 69 (75); krit im Hinblick auf das Kriterium ,good commercial practice“": Jansen, Klauselkontrolle, in Eidenmüller/Faust/Grigoleit/Jansen/Wagner/Zimmermann, Revision des Verbraucher-acquis 53 ( $74 \mathrm{ff}, 84 \mathrm{ff}$ ); siehe auch Jansen/Zimmermann, Grundregeln des bestehenden Gemeinschaftsprivatrechts?, JZ 2007, 1113 (1120 f).

${ }^{97}$ Mazeaud, Unfairness and Non-negotiated Terms, in Schulze/Stuyck, Towards a European Contract Law 123 (147).

${ }^{98}$ Das Kriterium des „Nichtaushandelns“ spielt in der Praxis eine eher geringfügige Rolle.

${ }^{99}$ Hier werden die skandinavischen Inspirationsquellen sichtbar; vgl hierzu Wilhelmsson, Standard Form Conditions, in Hartkamp/Hesselink/Hondius/Joustra/du Peron/Veldman, Towards a European Civil Code $^{3} 431$ (440).
} 
zu rasch aufgegeben wird. ${ }^{100}$ Das Modell der Studie scheint zu repressiv zu sein.

Nur für Verträge zwischen Unternehmern und Verbrauchern gilt Art 88, der das Versprechen optionaler Nebenleistungen erfasst. Es handelt sich um solche Nebenleistungen des Unternehmers, auf die der Verbraucher ausdrücklich verzichten muss, wenn er für sie nicht zahlen will. In einem solchem Fall können die Bestimmungen nur dann zur Geltung kommen, wenn der Verbraucher sie ausdrücklich angenommen hat, bevor er sich durch den Vertrag bindet. Art 88 wird zu einer Sonderregelung des Transparenzgebotes bzw. der überraschenden Klauseln. Er ist zu Recht auf die Verbraucherverträge beschränkt, was nicht heißt, dass derartige Klauseln in rein unternehmerischen Verträgen unbedenklich sind. Dort müssen sie aber nach einem flexibleren Maßstab von dem Richter kontrolliert werden.

\section{Erfüllung und Leistungsstörungen}

Die Vorschriften über Erfüllung und Leistungsstörungen werden typischerweise ,personenneutral“ formuliert. Sogar die Verbrauchsgüterkauf-RL enthält Vorschriften, die ihrem Inhalt nach einen Kern des allgemeinen Kaufrechts bilden könnten. Deswegen kann auch die organische Verwandtschaft der Verbrauchsgüterkauf-RL und des UN-Kaufrechts an vielen Stellen nachgewiesen werden. Der Hauptunterschied zwischen spezifischen und nicht spezifischen Verbrauchernormen liegt häufig in der zwingenden Natur der Ersteren. Bei den Unternehmern hingegen kommen häufig kurze Inspektions- und Mahnungsfristen hinzu.

Die Machbarkeitsstudie folgt diesem Modell an vielen Stellen. Die Mehrheit der Vorschriften wird ihrem Inhalt nach „personenneutral“ formuliert. Regelmäßig sind die Vorschriften, welche dem Schutz einer Partei dienen, für Verbraucherverträge zwingend. Die Studie regelt aber an erstaunlich vielen Fällen die Erfüllung und Leistungsstörung im Bereich des Verbraucherrechts auch inhaltlich abweichend. Das Recht der Unternehmer und das Recht der Verbraucher entfernen sich also in wichtigen Punkten voneinander.

\subsection{Vertragsmäßigkeit der Leistung}

Art $103^{101}$ und $105^{102}$ der Studie bestimmen Kriterien der Vertragsmäßigkeit der Leistung, von denen grundsätzlich nicht abgewichen werden kann, es sei denn, dass der Verbraucher zum Zeitpunkt des Vertragsschlusses die

\footnotetext{
${ }^{100}$ Ausführlich zu dieser Frage Zoll, Der Tod der Allgemeinen Geschäftsbedingungen (in Druck).

${ }^{101}$ Art 101 August-Version.

${ }^{102}$ Art 103 August-Version.
}

besonderen Eigenschaften der Ware kannte und sie als vertragsgemäß akzeptiert hat. ${ }^{103}$ Auf diese Weise wird versucht das Problem der sog. „negativen Beschaffenheitsvereinbarungen “ $\mathrm{zu}$ lösen. ${ }^{104}$ Der Unternehmer trägt also die Beweislast dafür, dass der Verbraucher in voller Kenntnis der Sachlage auf bestimmte, unter normalen Umständen zu erwartende Merkmale der Kaufsache verzichtet hat. Es reicht nicht aus, dass im Vertrag bestimmte Eigenschaften der Sache, die unter die Erwartungen aus Art 103 und 105 fallen, schlicht genannt werden. Ein positives Wissen und Einverständnis des Verbrauchers, dass er zum Zweck des Erwerbs von Sachen minderer Qualität absichtlich gehandelt hat, muss auf diese Weise klar zum Ausdruck gebracht werden

Praktisch führt die angenommene Lösung zu der Objektivierung der Vertragswidrigkeit, da der Schwerpunkt der Bestimmung der Vertragsmäßigkeit grundsätzlich in den typisch zu erwartenden Eigenschaften der Sache liegt und nicht in der getroffenen Vereinbarung. Theoretisch wird zwar die Kohärenz mit der subjektiven Konzeption der Vertragswidrigkeit dadurch erhalten, dass die Schwelle des darauf gerichteten wirksamen Konsenses sehr stark angehoben wird. Praktisch aber werden die objektiven Eigenschaften der Sache in Bezug auf die typischen Erwartungen maßgeblich. ${ }^{105}$

In Verbraucherverträgen ist diese Lösung akzeptabel. In grenzübergreifenden Verbrauchsgüterkaufverträgen kommt es zu einer an Massen orientierten Standardisierung und deswegen können auch typische Erwartungen eine entscheidende Rolle spielen. Der Verbraucher wird dann zusätzlich gegen den Verlust von Rechten geschützt. Würde der persönliche Anwendungsbereich des Instruments erweitert, sollte diese Konstruktion weiter auf die Verbraucher beschränkt bleiben, weil sie die Gestaltungsfreiheit für Verträge sehr stark einschränkt.

\subsection{Kenntnis von der Vertragswidrigkeit}

Nach Art $106^{106}$ haftet der Verkäufer für eine Vertragswidrigkeit dann nicht, wenn der Käufer zum Zeitpunkt des Vertragsschlusses die Vertragswidrigkeit kannte oder hätte

\footnotetext{
${ }^{103}$ Art 102 Abs 3 Machbarkeitsstudie (Art 100 Nr 3 August-Version).

${ }^{104} \mathrm{Zu}$ der Problematik negativer Beschaffenheitsvereinbarungen im deutschen Recht siehe Lorenz in MK-BGB III ${ }^{5}$ (2008) § 475 Rz 8, 9; Faust in Bamberger/Roth BGB ${ }^{20} \S 475 \mathrm{Rz} 8 \mathrm{ff}$; ausf hierzu auch Gsell, „Fehlerbegriff und (negative) Beschaffenheitsvereinbarungen“ im Tagungsband „European Law Days“ Osnabrück, 30.06.-01.07.2011 (in Druck).

${ }^{105}$ Zum subjektiven Fehlerbegriff siehe Westermann in MK-BGB III ${ }^{5}$ $\S 434$ Rz 5, 8; Saenger in Schulze ea, Bürgerliches Gesetzbuch ${ }^{6} \S 434$ Rz 1, 7; Riesenhuber, System und Prinzipien des Europäischen Vertragsrechts (2003) $480 \mathrm{f}$.

${ }^{106}$ Art 105 August-Version.
} 
kennen müssen. Diese Haftungseinschränkung gilt aber nur im Fall von Verträgen, die ausschließlich zwischen Unternehmern geschlossen werden. Das heißt, dass die Kenntnis des Verbrauchers von der Vertragswidrigkeit in Verbraucherverträgen nicht zum Ausschluss der sich aus der Leistungsstörung ergebenden Rechte führt.

Der Ausschluss der Gewährleistungsrechte im Fall der Kenntnis hat eine lange Geschichte. Fraglich ist, ob die Regel als solche sinnvoll ist, vor allem dann, wenn der Verkäufer bestimmte Eigenschaften der Ware ausdrücklich zugesichert hat. Die Berufung auf die Gewährleistungsrechte trotz der Kenntnis von der Vertragswidrigkeit der Ware sollte nicht als venire contra factum proprium qualifiziert werden. Es ist nicht überzeugend, dass ein Kunde, der weiß, dass der Versprechende sein Versprechen nicht einhalten wird, so gestellt wird, als wäre kein Versprechen gegeben worden. Im Fall neuer vertretbarer Sachen könnte häufig davon ausgegangen werden, dass die zu liefernde Sache noch entsprechend ergänzt und vervollständigt wird, bevor sie dem Kunden zugestellt wird. Die Kenntnis des Kunden kann in solchen Fällen nicht so gedeutet werden, dass der Käufer auf seine Rechte verzichten wollte. Im Regelfall würde eine solche Vermutung den typischen Motivationen eines rational handelnden Menschen widersprechen.

Abgesehen von der Frage, ob die Norm sachlich richtig ist, sollte sie gleich für alle Personen gelten - und zwar unabhängig von ihrem Status. Diese Norm ist entweder sachlich richtig für alle Konstellationen oder sie ist falsch für alle. Sie sollte vielleicht detaillierter sein. Bereits im römischen Recht galt eine solche Regel im Fall der ausdrücklichen Zusicherungen nicht. ${ }^{107}$

\subsection{Recht zur zweiten Andienung}

Die Machbarkeitsstudie regelt sehr detailliert das Recht des Verkäufers zur zweiten Andienung (right to cure $^{108}$ ), das dem Käufer die Geltendmachung von Rechtsbehelfen (mit Ausnahme des Zurückbehaltungsrechts) verwehrt, die für den Fall der Leistungsstörung vorgesehen sind. ${ }^{109}$ Dieses Privileg gilt aber nicht für Verbraucherverträge, mit Ausnahme

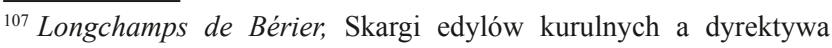
1999/44/EC Parlamentu Europejskiego i Rady w sprawie określonych aspektów sprzedaży i gwarancji na dobra konsumpcyjne „Studia Iuridica“ 44/2005, 427 (431 Pkt 2); Zimmermann, The Law of obligations (1996) 309.

${ }^{108}$ Näher zum Recht auf zweite Andienung siehe: Machnikowski/Szpu$n a r$, in Acquis Group, Acquis Principles II Provisional Comments zu Art 8:102; v. Bar/Clive, DCFR Full Edition Comments zu Art III.-3: 302.

${ }^{109}$ Art 110 Abs 1 Machbarkeitsstudie (Art 110 Nr 1 August-Version).
}

derer, die Dienstleistungen zum Gegenstand haben ${ }^{110}$ und der gemischten Verträge mit einem Dienstleistungsteil ${ }^{111}$.

Dies ist eine Entscheidung, die von dem Konzept der Verbrauchsgüterkauf-RL abweicht. ${ }^{112}$ In der Tat ist das Recht auf zweite Andienung für den Verbraucher häufig eine wesentliche Hürde bei der Geltendmachung seiner Rechte, vor allem dann, wenn der Kontakt mit dem Unternehmer erschwert ist. Im Fall grenzüberschreitender Verträge stellt die zusätzliche Notwendigkeit der Verwicklung in eine Rechtsbeziehung mit einer anderen Partei eine zusätzliche Hürde dar, die den Verbraucher häufig zum Verzicht auf die Geltendmachung der ihm zustehenden Rechte bewegen kann. Aus diesem Grund ist ein Verzicht auf dieses Recht des Verkäufers angesichts der Funktion des Optionalen Instruments eher zu begrüßen, zumindest wenn der Vertrag standardisierte, vertretbare Güter zum Gegenstand hat. Für Verträge, die Waren zum Gegenstand haben, die auf die individuellen Bedürfnisse des Kunden angepasst werden, ist diese Regel für den Verkäufer vielleicht zu restriktiv. In diesen Fällen ist der Unternehmer gleichermaßen schutzwürdig wie bei Verträgen über eine Dienstleistung. Das Recht zur zweiten Andienung wird aber in einer relativ käuferfreundlichen Version vorgesehen. Der Verkäufer muss durch sein Andienungsangebot vortreten. ${ }^{113}$ Diese sanftere Form wäre vielleicht auch für Verbraucherverträge angemessen, sofern der Gesetzgeber das Recht zur zweiten Andienung generell angemessen findet.

Die gleiche Interessenlage ist auch in dem Fall gegeben, in dem der Kunde Unternehmer ist. Würde es sich bei dem Vertragsgegenstand um Massenware handeln, die im Rahmen eines grenzüberschreitenden Geschäfts geliefert wird, ist ein derartiger Unterschied zwischen Unternehmern und Verbrauchern nicht zu rechtfertigen. Der unternehmerische Käufer kann mit den gleichen Schwierigkeiten konfrontiert werden, die einem Verbraucher in solchen Fällen begegnen können. Bei Massengeschäften, für die sich das Optionale Instrument vielleicht besonders eignet, wäre der kleine oder mittlere Unternehmer durch die Notwendigkeit, sich auf Rechtsbeziehungen einzulassen, die durch die zweite Andienung ausgelöst werden, häufig auch praktisch daran gehindert, seine Rechte durchzusetzen.

Bei Kaufverträgen kann es häufig vorkommen, dass der Kunde zum Teil zu einem privaten, zum Teil zu einem unternehmerischen Zweck handelt. Die Machbarkeitsstudie enthält keine ausdrückliche Regelung, die Verträge mit

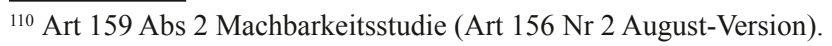
${ }^{111}$ Art 110 Abs 8 Machbarkeitsstudie (nicht mehr in der August-Version).

${ }^{112}$ Krit im Hinblick auf das in der Verbrauchsgüterkauf-RL statuierte Verbraucherschutzniveau siehe: Pecyna, Ustawa o sprzedaży konsumenckiej (2007) Art 8 ust 1, 162, 163 mwN; auch Pisuliński, in Rajski, System Prawa Prywatnego VII $\S 14$ Rz 485 und 490.

${ }^{113}$ Art 110 Abs 1 Machbarkeitsstudie (Art 110 Nr 1 August-Version).
} 
einem gemischten privat-unternehmerischen Zweck erfassen würde. Eine derartige Lücke schadet nur dann, wenn das Verbraucherregime sich wesentlich von der allgemeinen bzw. unternehmerischen Vertragsordnung unterscheidet. Dann kommt es zu praktischen Problemen sowie zu Wertungskollisionen. Ähnliche Situationen sollten demnach ganz unterschiedlich entschieden werden. Welche Regeln angewendet werden, ist kaum vorherzusehen. Die Verfasser der Machbarkeitsstudie sollten solche Situationen vermeiden, in welchen mit nicht leicht feststellbaren Kriterien zu weitgehende Unterschiede verbunden werden. Es ist nicht einfach in einem grenzüberschreitenden, häufig elektronischen Rechtsverkehr den persönlichen Status der anderen Partei zu ermitteln. In Verträgen mit einem gemischten Zweck ist dies noch schwieriger. Aus diesem Grund könnte eine wesentliche Rechtsunsicherheit entstehen: Ist ein Rücktritt wirksam oder wird er durch die Ausübung des Rechtes zur zweiten Andienung aufgeschoben?

Die bestehenden Unterschiede im System des Rechts zur zweiten Andienung zwischen dem Verbraucher- und Unternehmerregime sind in der Machbarkeitsstudie unnötig. Die unterschiedliche Betrachtung findet keine fundamentale Rechtfertigung in der Interessenlage der Parteien, bringt aber eine unnötige Erschwerung in der möglichen Funktionsweise des Optionalen Instruments mit sich.

Würde der persönliche Anwendungsbereich des Optionalen Instruments erweitert und die bestehenden Unterschiede aufrechterhalten, wäre es vielleicht besser, das Verbraucherregime in dieser Hinsicht um diese zusätzlichen Konstellationen zu erweitern. Zwischen Privaten - vor allem im elektronischen Handel - könnte das Recht zur zweiten Andienung ein wesentliches Hindernis für die Geltendmachung der Rechte des Käufers sein, da es noch schwieriger ist, den Verkäufer wirksam zu erreichen. Die zusätzliche Verwicklung in die Reparatur bzw. Nacherfüllung könnte für den Käufer mit übermäßigen Belastungen verbunden sein. ${ }^{114}$ Welches Regime anwendbar ist im Fall der Kunden, die weder Unternehmer noch Verbraucher sind, wäre eine durch einen Zufall bedingte Entscheidung des Gesetzgebers. Für die Differenzierung beider Regime gibt es keine ausreichenden Gründe. Deswegen ist es in Grenzfällen schwieriger, die Wertungen aufzuzeigen, die für diese bzw jene gesetzgeberische Entscheidung sprechen würden.

\subsection{Prüfungs- und Rügeobliegenheiten in Unternehmerverträgen}

Nach dem Vorbild des CISG $^{115}$ wird in den Unternehmerverträgen eine Obliegenheit zur Überprüfung und Rüge der

\footnotetext{
${ }^{114}$ Siehe Fn 101.

${ }^{115}$ Art 39 CISG; im Unterschied zum CISG und der in der Machbarkeitsstudie getroffenen Regelung ist Art IV.A.-4:301 DCFR nicht unternehmerspezifisch ausgestaltet.
}

Vertragswidrigkeit unter Wahrung einer angemessenen Frist zur Anzeige der Vertragswidrigkeit vorgesehen. ${ }^{116}$ Unabhängig von der von F. Faust erhobenen Kritik, die Regelung der Überprüfungspflicht sei nicht erforderlich ${ }^{117}$, weil allein die Notwendigkeit der Rüge ausreichen würde, ist dies eine typische Norm, die im Unternehmerbereich der Disziplinierung der Parteien dient und unnötige Rechtsunsicherheit beseitigt. Würde der persönliche Anwendungsbereich des Instruments erweitert, sollte diese Norm weiter nur für Unternehmer gelten. Von anderen Parteien kann eine derartige Disziplin zur Wahrung eigener Interessen nicht erwartet werden. Die einschlägigen Verjährungsfristen sind als ausreichend anzusehen.

\subsection{Zuviellieferung in Verbraucherverträgen}

Die Fragen der Zuviellieferung werden in der Machbarkeitsstudie in Art 132 geregelt. ${ }^{118}$ Die Formulierung dieser Regelung ist personenneutral. Im Fall der Zuviellieferung steht der Käufer von der Wahl, die nicht geschuldete Mehrleistung zu verweigern oder anzunehmen. ${ }^{119}$ Entscheidet er sich für die zweite Möglichkeit, muss er die vertraglich vereinbarte Vergütung bezahlen. ${ }^{120}$ Für Verbraucher gilt aber eine Modifikation. Hat der Verbraucher vernünftige Gründe zu glauben, dass die Mehrlieferung vorsätzlich, ohne Irrtum auf Seiten des Verkäufers erfolgte, findet die Vorschrift über die Zusendung unbestellter Waren Anwendung, was dazu führt, dass der Verbraucher die Sache behalten kann und ihn keine Kosten und Zahlungspflichten treffen. ${ }^{121}$ Der Gedanke des erweiterten Verbraucherschutzes in diesem Fall ist zu begrüßen, da hier tatsächlich Gründe vorliegen, die mit den Gründen für den Verbraucherschutz bei unbestellten Lieferungen im Sinne von Art 39 vergleichbar sind. Die Vorschrift ist allerdings unscharf formuliert und die Feststellung, unter welchen Umständen die Überzeugung des Verbrauchers über den Vorsatz der Mehrlieferung ,vernünftig“" war, ist allein dem Richter überlassen, was ein zusätzlichen Unsicherheitsfaktor darstellt. Das ist wiederum ein zusätzliches Risiko für den Unternehmer, da ihm Vorsatz nicht nachgewiesen werden muss. Vielmehr genügt die vernünftige Überzeugung des Verbrauchers über das Bestehen einer solchen Absicht.

\footnotetext{
${ }_{116}$ Art 123 und 124 Machbarkeitsstudie (Art 122 und 123 AugustVersion); diese Regelung hat sich in vielen Zivilrechtskodfikationen durchgesetzt, siehe Ranieri, Europäisches Obligationenrecht ${ }^{3}$ (2009) $867 \mathrm{f}$.

${ }^{117}$ So Faust in Tagungsband „European Law Days“ (Fn 104).

${ }^{118}$ Vgl Art 52 Abs 2 CISG und Art IV. A.-3:105 DCFR.

${ }^{119}$ Art 132 Abs 3 Machbarkeitsstudie (Art 131 Nr 3 August-Version).

${ }^{120}$ Art 132 Abs 4 Machbarkeitsstudie (Art 131 Nr 4 August-Version).

${ }^{121}$ Art 132 Abs 5 iVm Art 39 Machbarkeitsstudie (Art 131 Nr 5 August-Version).
} 


\subsection{Zahlungsverzug bei Verbraucherverträgen}

Die Machbarkeitsstudie sieht in Art 172 besondere Regelungen vor, die den Verbraucher im Fall seiner verspäteten Zahlung vor zu hohen Zinsen schützen sollen. Dieser Schutz geht erstaunlich weit. Der Verbraucher hat nur dann Zinsen zu zahlen, wenn er die Verspätung zu vertreten hat (Abs 1). Zusätzlich werden die Zinsen nicht für die ersten 30 Tage nach der Warnung des Gläubigers berechnet, in welcher er die Zinsen bestimmt und zu deren Zahlung auffordert. Darüber hinaus ist die Zinsenhöhe nach Art 171 sehr niedrig, wodurch der Schuldner und insbesondere der Verbraucher im Fall eines Zahlungsverzugs übermäßig geschützt werden. ${ }^{122}$ Das sind zu starke Anreize, nicht zu zahlen, insbesondere auch, weil in vielen Ländern die Kredite wesentlich teurer sind als diese „Strafzinsen“. Das Verbraucherschutzrecht sollte keine Anreize für eine Vertragsverletzung schaffen. Es ist kaum vorstellbar, dass diese Vorschriften in einer solchen Form aufrechterhalten bleiben. Hierdurch werden die Unternehmer von der Wahl des Instruments wirksam abgehalten. ${ }^{123}$

\subsection{Zahlungsverzug im unternehmerischen Bereich}

Die Studie übernimmt wesentliche Elemente der Zahlungsverzugs-RL. ${ }^{24}$ Eine Einschränkung auf Unternehmer ist in diesem Fall berechtigt, weil es sich hierbei um ein spezifisches kaufmännisches Problem handelt.

\section{Vereinbarungen über die Verjährung}

Nach Art 189 Abs 4 sind die Vorschriften über die Verjährung für Verbraucherverträge zwingend, dh eine Abweichung zum Nachteil des Verbrauchers ist unzulässig. Da auch die allgemeinen Regeln einer Modifikation der Verjährung Schranken setzen (Art 189 Abs 2 und 3), wird eine Erweiterung des persönlichen Anwendungsbereiches keine Schwierigkeiten bewirken.

\section{Schlussfolgerungen}

Grundsätzlich könnte die Erweiterung des Anwendungsbereichs des Entwurfes auf andere Personenbeziehungen als nur Unternehmer-Verbraucher und Unternehmer-Unterneh-

\footnotetext{
${ }_{122}$ Dies hat Basedow zutreffend im Rahmen der Diskussion bei der Konferenz „Towards a European Contract Law“ am 03.06.2011 in Leuven angemerkt.

${ }^{123}$ So auch bereits Basedow in Leuven (Fn 122).

${ }^{124}$ Art 3 Zahlungsverzug-RL 2000 und Art 3 Zahlungsverzug-RL 2011.
}

mer mühelos durchgeführt werden. Die Mehrheit der Vorschriften ist als Teil des allgemeinen Privatrechts formuliert und nur relativ geringe Veränderungen wären erforderlich, um diesen Zweck zu erreichen. Nur im Bereich der Leistungsstörung kommt es zu einer übermäßigen Zersplitterung der Erfüllungs- und Haftungsordnung in unterschiedlichen persönlichen Konstellationen. Die Kluft zwischen beiden Ordnungen ist ohne eine ausreichende Rechtfertigung zu groß. Das bedarf bei der Erweiterung des persönlichen Anwendungsbereiches weiterer Interventionen. Diese scheinen aber ohnehin erforderlich zu sein. Würde der europäische Gesetzgeber bei dem derzeitigen Anwendungsbereich bleiben, würde die Anwendbarkeit des Optionalen Instruments darunter unnötig leiden ${ }^{125}$, weil bei Kaufverträgen und damit verbundenen Verträge eine eindeutige Qualifikation und Zuordnung der Gegenpartei sehr häufig schwer ist. Daraus entsteht eine Unsicherheit darüber, ob die Wahl des Instruments wegen der unklaren Qualifikation der Gegenpartei wirksam ist. Das könnte zur Folge haben, dass das Instrument auch dort nicht gewählt werden würde, wo man dies nach seinem Anwendungsbereich könnte und es für die Parteien von Nutzen wäre. Das Beharren auf enge Anwendungsbereiche wäre nur dann zu rechtfertigen, wenn die Erweiterung des persönlichen Anwendungsbereiches eine weitgehende Verkomplizierung der Regelungen herbeiführen würde. ${ }^{126}$ Schließlich erlaubt die Tatsache, dass es sich vorliegend um ein fakultatives Regime handelt, Parteien, für welche diese Regeln unpassend sind, sich gegen diese Wahlmöglichkeit zu entscheiden und das Optionale Instrument nicht zu wählen. ${ }^{127}$ Die vorliegende Studie zeigt, dass der enge Anwendungsbereich des Vorschlags für ein Optionales Instrument nicht in der Natur seiner Normen gründet, sondern vielmehr Folge externer politischer Entscheidungen und Zurückhaltungen ist ${ }^{128}$.

\footnotetext{
${ }^{125}$ So auch Doralt, der von einem fundamentalen Fehler spricht, welcher das ganze Projekt in Frage stellen könnte (Fn 9) 1 (13; auch 17, 18).

${ }^{126}$ Doralt (Fn 9) 1 (14) und Augenhofer, A European Civil Law - for Whom and What Should it Include? Reflections on the Scope of Application of a Future European Legal Instrument, ERCL 2011, 195 (201-208), ziehen auch andere Rechtfertigungsgründe für einen engen (persönlichen) Anwendungsbereich in Erwägung und lehnen diese mit überzeugender Begründung ab.

${ }^{127} \mathrm{Zu}$ diesem Schluss kommt auch Doralt (Fn 9) 1 (18).

${ }^{128}$ Vgl hierzu auch Augenhofer (Fn 125) 195 (203).
} 